\title{
Dynamical Revivals in Fermi Accelerator Model
}

\author{
Farhan Saif \\ Department of Electronics, Quaid-i-Azam University, Islamabad, Pakistan.
}

(November 20, 2018)

\begin{abstract}
We investigate quantum revivals in the dynamics of an atom in an atomic Fermi accelerator. It is demonstrated that the external driving field influences the revival time significantly. Analytical expressions are presented which are based on semiclassical secular theory. These analytical results explain the dependence of the revival time on the characteristic parameters of the problem quantitatively in a simple way. They are in excellent agreement with numerical results.
\end{abstract}

PACS numbers: 03.65.Bz,05.45,72.15Rn,42.50.Lc,05.30Ch

Periodically driven quantum systems have received considerable attention over the past few years due to the presence of Anderson-like localization [1]. Some recent numerical work indicates the presence of quantum revivals [2] in periodically driven quantum systems [3,4] as well as in other two-degree-of-freedom systems [5]. Though many aspects of quantum localization are well understood by now, so far even the most elementary questions concerning revival effects in periodically driven quantum systems are not yet comprehensible. Under which circumstances do such revival phenomena appear and how do these revival effects depend on the frequency and on the driving amplitude of the applied periodic force? The main intention of this letter is to address these questions by considering a physical system which is of particular interest in the field of atom optics [6 8], namely an atom in an atomic Fermi accelerator. It is made up of an atomic mirror [9] modulated by means of a external periodic field [10,11]. It will be shown that quantum revivals can be observed in this physical system and that the dominant influence of the periodic driving force results in a change of the revival time. Simple analytical results are presented which explain the quantitative dependence of the revival time on the driving frequency and the strength of the driving amplitude. These analytical results are in excellent agreement with numerical results.

Let us consider an atom in a periodically driven gravitational cavity [9]. The atom moves under the influence of gravity in the positive $\tilde{z}$-direction and is reflected back as it hits a mirror, as shown in Fig 1. The atomic mirror is assumed to be made up of an evanescent electromagnetic field with electric field strength $\mathbf{E}(\tilde{z}, t)=\mathbf{e}_{x} E_{0} e^{-\omega_{L} \tilde{z} / c} e^{-i \omega_{L} t}+$ c.c. In order to study the effect of an external driving force on this elementary quantum system the evanescent wave is assumed to be modulated by an acusto-optical modulator [10], that is $E_{0}=\mathcal{E}_{0} \exp (\epsilon \sin \omega t)$. Assuming that the optical driving frequency $\omega$ is well detuned from any atomic resonance and taking into account the symmetry of the problem in the $(x, y)$-plane the effective one-dimensional centerof-mass motion of the atom is governed by the Hamilto- nian 11

$$
H=\frac{p^{2}}{2 M}+M g \tilde{z}+\frac{\hbar \Omega_{e f f}}{4} e^{-2 \omega_{L} \tilde{z} / c+\epsilon \sin \omega t} .
$$

Here $p$ is the atomic center-of-mass momentum, $M$ is the atomic mass and $g$ denotes the gravitational acceleration. The effective Rabi frequency $\Omega_{e f f}$ characterizes the strength of the influence of the applied electric field.

The potential generated by the gravitational acceleration and by the evanescent laser field has the approximate form of a time dependent triangular potential well like in the Fermi accelerator [12]. Thus, in the subsequent, approximate treatment we replace this potential by the idealized, simpler form of a triangular well with an infinitely high potential barrier at the position $\tilde{z}=\frac{c \epsilon}{2 \omega_{L}} \sin \omega t$ of the mirror. In this approximation our results become independent of details of the evanescent laser field, such as the laser frequency $\omega_{L}$ and the value of the effective Rabi frequency $\Omega_{e f f}$.

In the moving coordinate we may write the time dependent Schrödinger equation as 13

$$
i \hbar \dot{\chi}=\left\{\frac{p^{2}}{2 M}+M z\left(g-\lambda \omega^{2} \sin \omega t\right)+V_{0} e^{-\kappa z}\right\} \chi,
$$

where $z=\tilde{z}-\lambda \sin \omega t$. Here we are considering $V_{0} \equiv$ $\hbar \Omega_{\text {eff }} / 4$, the steepness $\kappa \equiv 2 \omega_{L} / c$ and the modulation strength $\lambda \equiv c \epsilon /\left(2 \omega_{L}\right)$.

A convenient way of obtaining insight into the influence of the acusto-optical external driving force on the atomic dynamics is to investigate the time evolution of an atomic center-of-mass wave packet. For this purpose we consider a Gaussian wave packet $\psi(0)=\left(2 \pi \Delta z^{2}\right)^{-1 / 4} \exp \{-(z-$ $\left.\left.z_{0}\right)^{2} /(2 \Delta z)^{2}\right\} \exp \left(-i p_{0}\left(z-z_{0}\right) / \hbar\right)$ at $t=0$ and propagate it in the gravitational cavity for different modulation strength $\lambda$. Here $z_{0}$ describes the average position, $p_{0}$ denotes the average momentum of the wave packet and $\Delta z$ is the spatial uncertainty. In Fig. 2 characteristic time dependences of the autocorrelation function $\mathcal{C}(t) \equiv\langle\psi(0) \mid \psi(t)\rangle$ of the wave packet are shown for $z_{0}=20.1 \mu m, \Delta z=0.28 \mu m$ and $p_{0}=0$. Without external periodic perturbation (uppermost figure) the well known scenario of revivals and fractional revivals [14] is 
clearly apparent. In the presence of a sufficiently weak external periodic driving force the revivals and fractional revivals are still observable. However, the revivals decrease in magnitude and the revival time exhibits a pronounced dependence on the external driving force.

We 113] calculate the revival time with the help of semiclassical secular theory. In the vicinity of the $N$-th primary resonance the classical Hamiltonian corresponding to Eq. (2) can be expressed in action and angle variables $(I, \varphi)$ [15] as

$$
\begin{gathered}
H=\frac{H^{\prime \prime}}{2}\left(I-I_{0}\right)^{2}+H^{\prime}\left(I-I_{0}\right) \\
+H_{0}\left(I_{0}\right)+\lambda V \sin (N \varphi-\omega t) .
\end{gathered}
$$

Here, $I_{0}$ is the classical action associated with the initial condition and $V=(M g)\left(I_{0} / I_{N}\right)^{2 / 3}$. The classical action at the center of the $N$-th primary resonance is denoted by $I_{N}$. Moreover $H^{\prime}$ and $H^{\prime \prime}$ are the first and second derivatives of energy with respect to action calculated at $I_{0}$. Similarly $H_{0}\left(I_{0}\right)$ is the energy of the unperturbed system for the initial action $I_{0}$. In Eq. (3) we have averaged out the fast oscillating terms so that $H\left(I-I_{0}\right)$ represents an integrable one-degree-of-freedom physical system.

Introducing the transformation $N \varphi-\omega t=2 \theta+\pi / 2$ and quantizing the dynamics around the $N$-th resonance by using $I-I_{0}=\frac{\hbar}{i} \frac{\partial}{\partial \varphi}=\frac{N \hbar}{2 i} \frac{\partial}{\partial \theta}[17$, the time independent Schrödinger equation becomes

$$
\begin{array}{r}
{\left[-\frac{N^{2} H^{\prime \prime} \hbar^{2}}{8} \frac{\partial^{2}}{\partial \theta^{2}}+\frac{\hbar}{2 i}\left(N H^{\prime}-1\right) \frac{\partial}{\partial \theta}+H_{0}\left(I_{0}\right)\right.} \\
+\lambda V \cos 2 \theta] \psi=\mathcal{E}_{n} \psi .
\end{array}
$$

Thus the quasi energies $\mathcal{E}_{n}$ are determined by Eq. (4). Here $\psi(\theta)$ has to fulfill the periodic boundary condition. It is straightforward to write Eq. (4) in the form of a Mathieu equation by substituting $\psi=$ $\phi \exp \left(-2 i\left(N H^{\prime}-1\right) \theta /\left(N^{2} H^{\prime \prime} \hbar\right)\right)$, namely

$$
\left[\frac{\partial^{2}}{\partial \theta^{2}}+a-2 q \cos 2 \theta\right] \phi=0,
$$

with

$$
\begin{aligned}
& a=\frac{8}{N^{2} H^{\prime \prime}\left(I_{0}\right) \hbar^{2}}\left[\mathcal{E}_{n}-H_{0}\left(I_{0}\right)+\frac{\left(N H^{\prime}\left(I_{0}\right)-1\right)^{2}}{2 N^{2} H^{\prime \prime}\left(I_{0}\right)}\right], \\
& q=\frac{4 \lambda V}{N^{2} H^{\prime \prime}\left(I_{0}\right) \hbar^{2}}
\end{aligned}
$$

The quasi-energy eigenvalues $\mathcal{E}_{n}$ are determined by the solutions of Eq. (5) and the requirement that $\phi(\theta+\pi)=$ $\phi(\theta)$. The $\pi$-periodic solutions of Eq. (5) correspond to even functions of the Mathieu equation whose corresponding eigenvalues are real [18]. These solutions are $\phi_{\nu}(\theta)=e^{i \nu \theta} P_{\nu}(\theta)$ where $P_{\nu}(\theta)$ is the even order Mathieu function. In order to obtain a $2 \pi$-periodic solution in $\varphi$-coordinate we require the coefficient of $\varphi$ to be equivalent to an integer number. This requirement provides us the oppertunity to find the value for the index $\nu$ of the Mathieu functions as

$$
\nu=\frac{2}{N \hbar}\left[I-4 I_{0}+3 I_{0}\left(\frac{I_{0}}{I_{N}}\right)^{1 / 3}\right],
$$

where $I=(n+3 / 4) \hbar$. The quasi energy of the system is finally given by

$$
\mathcal{E}_{n}=\frac{N^{2} H^{\prime \prime} \hbar^{2}}{8} a_{\nu(n)}(q)-\frac{\left(N H^{\prime}-1\right)^{2}}{2 N^{2} H^{\prime \prime}}+H_{0}\left(I_{0}\right),
$$

with the Mathieu characteristic parameter $a_{\nu(n)}(q)$.

In order to check this result we study case of zero modulation strength, that is $\lambda=0$ which implies $q=0$. In this case the value for Mathieu characteristic parameter becomes $a_{\nu}(q=0)=\nu^{2}$. This leads us to Hamiltonian corresponding to schrödinger equation of Eq. 3 in the absence of external modulation.

We [13] employ the quasi-energy spectrum $\mathcal{E}_{n}$ of the driven system to evaluate the revival time. This concept helps us to define the time of revival $T_{\lambda}$ for time dependent system as

$$
T_{\lambda}=4 \pi \hbar\left(\left|\frac{\partial^{2} \mathcal{E}_{n}}{\partial n^{2}}\right|_{n=n_{0}}\right)^{-1} .
$$

From the quasi-energies of Eq. (9) we can determine the revival time in the presence of the external time dependent field. Considering $q<1$ we expand the Mathieu characteristic parameter $a_{\nu(n)}(q)$ up to $q^{2}$. We simplify our result by noting that $H^{\prime \prime}=-\left(M g^{2}\right)^{1 / 3}\left(\pi / 9 I_{0}^{2}\right)^{2 / 3}$ and $N=\left(\omega^{3} / M g^{2}\right)^{1 / 3}\left(3 I_{N} / \pi^{2}\right)^{1 / 3}$. Thus we finally obtain

$$
T_{\lambda}=T_{0}\left[1-\frac{1}{2}\left\{\frac{8 \lambda}{\hbar^{2}} E_{N}\left(\frac{I_{0}}{I_{N}}\right)^{2}\right\}^{2} \frac{3 \nu^{2}+1}{\left(\nu^{2}-1\right)^{3}}\right] .
$$

On substituting the value of $\nu$, calculated at $I=I_{0}$, in Eq. (11) we get

$$
T_{\lambda}=T_{0}\left[1-\frac{1}{8}\left\{\frac{M \lambda g}{E_{n_{0}}}\right\}^{2} \frac{3(1-r)^{2}+a^{2}}{\left((1-r)^{2}-a^{2}\right)^{3}}\right]
$$

where $r \equiv\left(E_{N} / E_{n_{0}}\right)^{1 / 2}$ and $a \equiv r^{2} \hbar \omega / 4 E_{n_{0}}$. If the initial energy is large, i.e. $E_{n_{0}} \gg \hbar \omega$, we may consider $a^{2}$ much smaller than $(1-r)^{2}$, which leads to

$$
T_{\lambda}=T_{0}\left[1-\frac{3}{8}\left\{\frac{M \lambda g}{E_{n_{0}}}\right\}^{2} \frac{1}{(1-r)^{4}}\right] .
$$

Our analytical result explain in a simple way the quantitative dependence of the revival time $T_{\lambda}$ on the characteristic parameters of the problem, namely the driving 
frequency $\omega$ and the driving amplitude $\lambda$. In order to access the accuracy of these perturbative results we calculate the revival time $T_{\lambda}$ by integrating the Schrödinger equation Eq. (2) numerically, and compare it with the analytically obtained result of Eq. (13). For this comparison we have considered two different initial conditions of the atomic wave packet above the surface of the atomic mirror. In Fig. 3 solid lines with circles corresponds to $(a) z_{0}=29.8 \mu \mathrm{m}$ which implies a state with mean principle quantum number $n_{0}=322.51$, however, solid line with squares corresponds to $(b) z_{0}=20.1 \mu \mathrm{m}$ which implies $n_{0}=176.16$. In both cases initial average momentum $p_{0}=0$. The first initial condition lies further away from the center of the corresponding primary resonance as compared to the second one.

Numerically we find that the change in revival time depends quadratically on the strength of external modulation $\lambda$ as predicted from Eq. (13). We plot the analytically obtained result for the two initial condition in Fig. 3 with the help of dashed lines. We find that the change of the revival time is smaller in the case $(a)$ than in the case $(b)$. Our analytical result explain this dependance: In case (a) of Fig. 3 our chosen initial condition $z_{0}=29.8 \mu m$ has a higher energy $E_{n_{0}}$ than in case (b) where $z_{0}=20.1 \mu \mathrm{m}$. Since the change in revival time has inverse dependence on the square of the energy $E_{n_{0}}$, as a result, we observe a smaller change in revival time when the average energy is larger as in case $(a)$ and a larger change in revival time when the average energy is smaller as in $(b)$.

We have demonstrated that the dynamics of a material wave packet in a periodically driven gravitational cavity exhibits quantum mechanical revivals. We have presented the first results on the quantitative dependence of the revival time on the characteristic parameters of the problem, namely the driving frequency and the driving strength. We show that these dependences can be understood quantitatively in a satisfactory way by using semiclassical secular theory. In view of the recent experimental [10] developments the presented quantitative predictions are accessible to experimental observation.

We thank G. Alber, I. Bialynicki-Birula, M. Fortunato, M. El Ghafar, R. Grimm, V. Savichev, P. Törmä, W. P. Schleich and A. Zeiler for many fruitful discussions. The author convays his special thanks to G. Alber for careful look on manuscript.

[1] For a review see M. G. Raizen, in Advances in Atomic, Molecular, and Optical Physics Vol. 41, p. 41 ed. B. Bederson and H. Walther (Academic Press, New York 1999).

[2] T. Hogg and B. A. Huberman, Phys. Rev. Lett. 48, 711 (1982).

[3] F. Haake, Quantum Signatures of Chaos, (Springer, Berlin 1992).

[4] J. K. Breslin, C.A. Holmes, and G.J. Milburn, Phys. Rev. A 56, 3022 (1997).

[5] S. Tomsovic and J. Lefebvre, Phys. Rev. Lett. 79, 3629 (1997).

[6] For a review of the field of atom optics see the special issue, J. Mlynek, V. Balykin, and P. Meystre, Special issue on Optics and Interferometery with Atoms, Appl. Phys. B 54, 319 (1992).

[7] P. Pillet, Special issue on Optics and Interferometry with Atoms, J. Phys. II 4, 1877 (1994);

[8] E. Arimondo and H.A. Bachor, (eds.), Special issue on Atom Optics, J. Quant. Semicl. Opt. 8, 495 (1996).

[9] H. Wallis, J. Dalibard, and C. Cohen-Tannoudji, Appl. Phys. B 54, 407 (1992).

[10] A. Steane, P. Szriftgiser, P. Desbiolles and J. Dalibard, Phys. Rev. Lett. 74, 4972 (1995).

[11] F. Saif, I. Bialynicki-Birula, M. Fortunato, and W.P. Schleich, Phys. Rev. A 4779, 581998.

[12] E. Fermi, Phys. Rev. 75, 1169 (1949).

[13] For a comprehensive study of revival phenomena in driven system, see, F. Saif, Ph.D thesis, (Universität Ulm, 1998).

[14] I. Sh. Averbukh and N.F. Perel'man, Phys. Lett. A 139, 449 (1989).

[15] A.J. Lichtenberg and M.A. Lieberman, Regular and Stochastic Motion, (Springer, Berlin, 1983) and references therein.

[16] L.D. Landau and E.M. Lifschitz, Quantenmechanik, (Akademie-Verlag, Berlin, 1971).

[17] M. V. Berry, Philos. Trans. R. Soc. London, Ser. B 287, 237 (1977).

[18] M. Abramowitz and I.A. Stegun, Handbook of Mathematical Functions, (Dover, New York, 1992). 
FIG. 1. Experimental setup of atomic Fermi accelerator: Cloud of cesium atoms is trapped and cooled in a magneto optical trap (MOT) down to the micro-Kelvin scale. The MOT is placed at a certain height above the evanescent wave mirror. The mirror for the atoms results due to the total internal reflection of the laser light field from the surface of the glass prism. The atomic mirror is modulated by providing an intensity modulation to the evanescent light field via an acousto optic modulator. The polarization of the evanescent wave field is inside the plane of the reflection. We consider the atomic dynamics only along the $z$-axis, that is, along the normal to the surface of the mirror.

FIG. 2. Revival phenomena in a gravitational cavity in the absence and in the presence of periodic modulation: Autocorrelation of a Gaussian wave packet of a cesium atom as a function of time, prepared at $t=0$ with $\Delta z=0.28 \mu m$, for $\lambda=0$ (a), $\lambda=0.56 \mu m$ (b), $\lambda=1.13 \mu m$ (c), and $\lambda=2.26 \mu m$ (d). The parameters are $\omega=2 \pi \times 0.93 \mathrm{KHz}, \Omega_{\text {eff }}=23.38 \mathrm{KHz}$, and $\kappa^{-1}=0.57 \mu \mathrm{m}$. The average position of the wave packet in the gravitational cavity is $z_{0}=20.1 \mu \mathrm{m}$ which corresponds to the mean quantum number $n_{0}=176.16$.

FIG. 3. We compare the ratio between revival time for driven system and revival time of undriven system, $T_{\lambda} / T_{0}$, for two different initial conditions, $z_{0}=29.8 \mu \mathrm{m}$ (solid line with circles) and $z_{0}=20.1 \mu \mathrm{m}$ (solid line with squares). The comparison comes from exact numerical calculations of time dependent Schrödinger equation Eq. (2) (solid lines), and from analytical results based on Eq. (12) (dashed lines). All the other parameters are the same as in Fig. 2. 


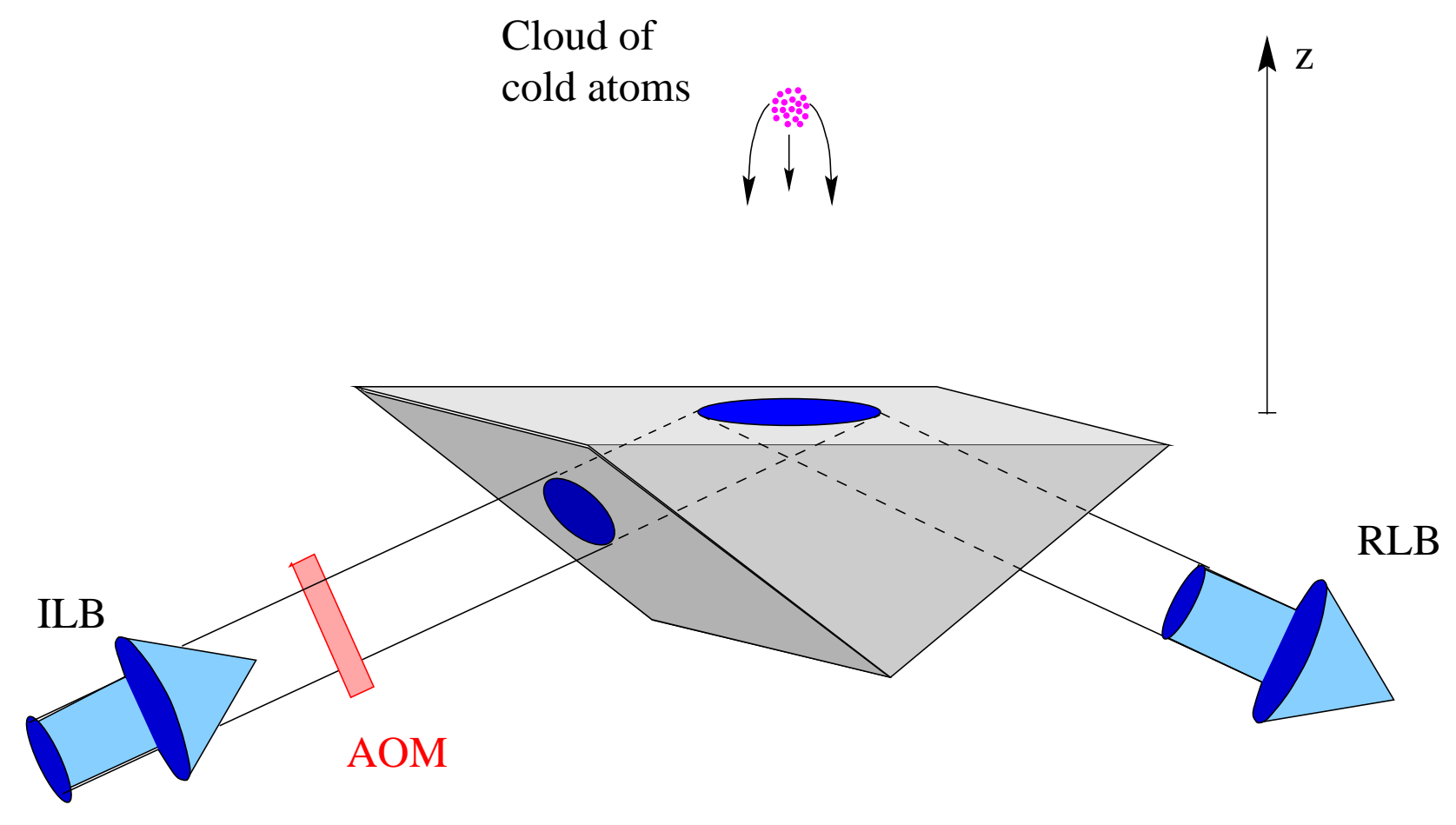

ILB: Incident Laser Beam

RLB: Reflected Laser Beam

AOM: Acousto Optic Modulator 


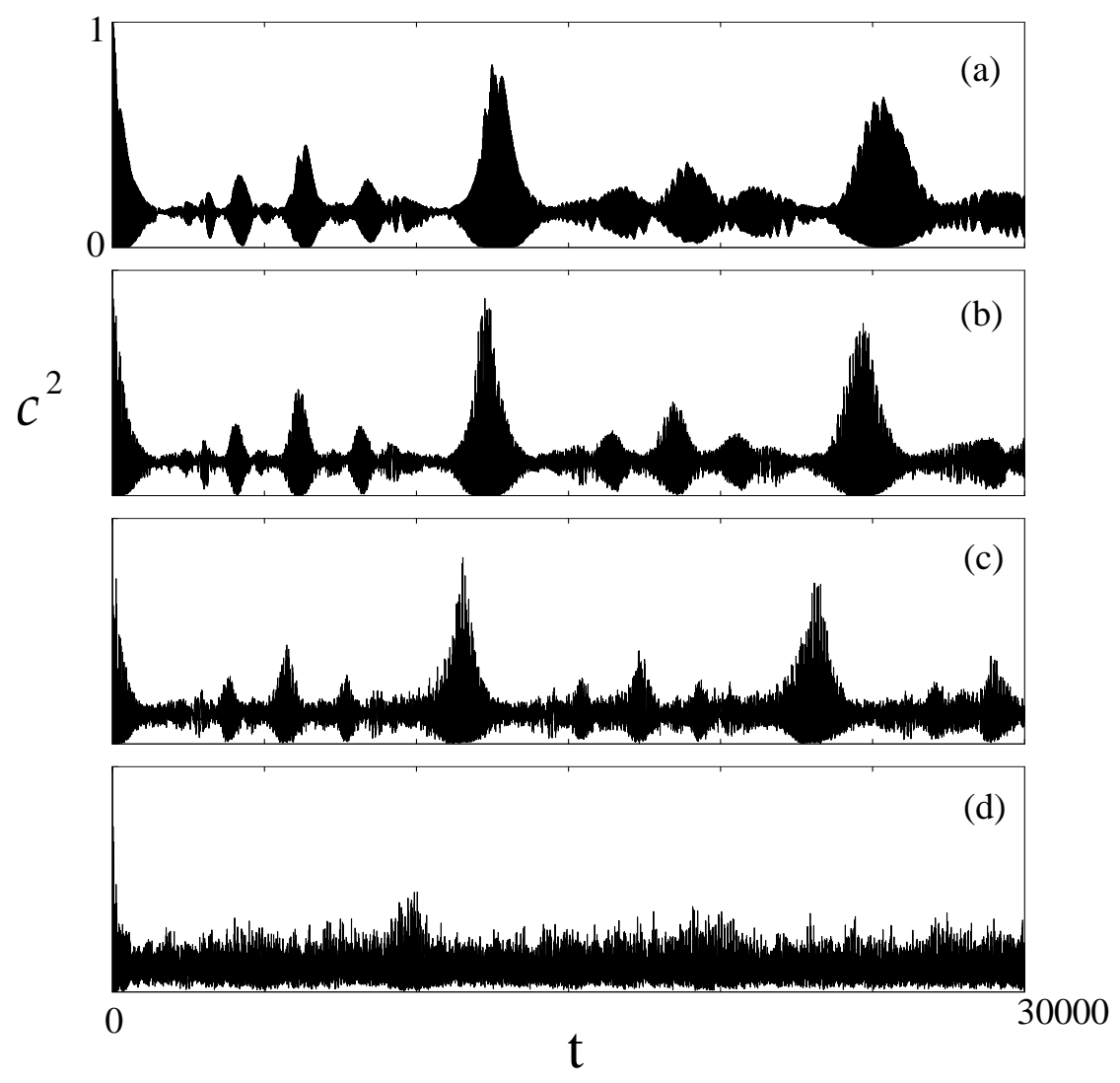




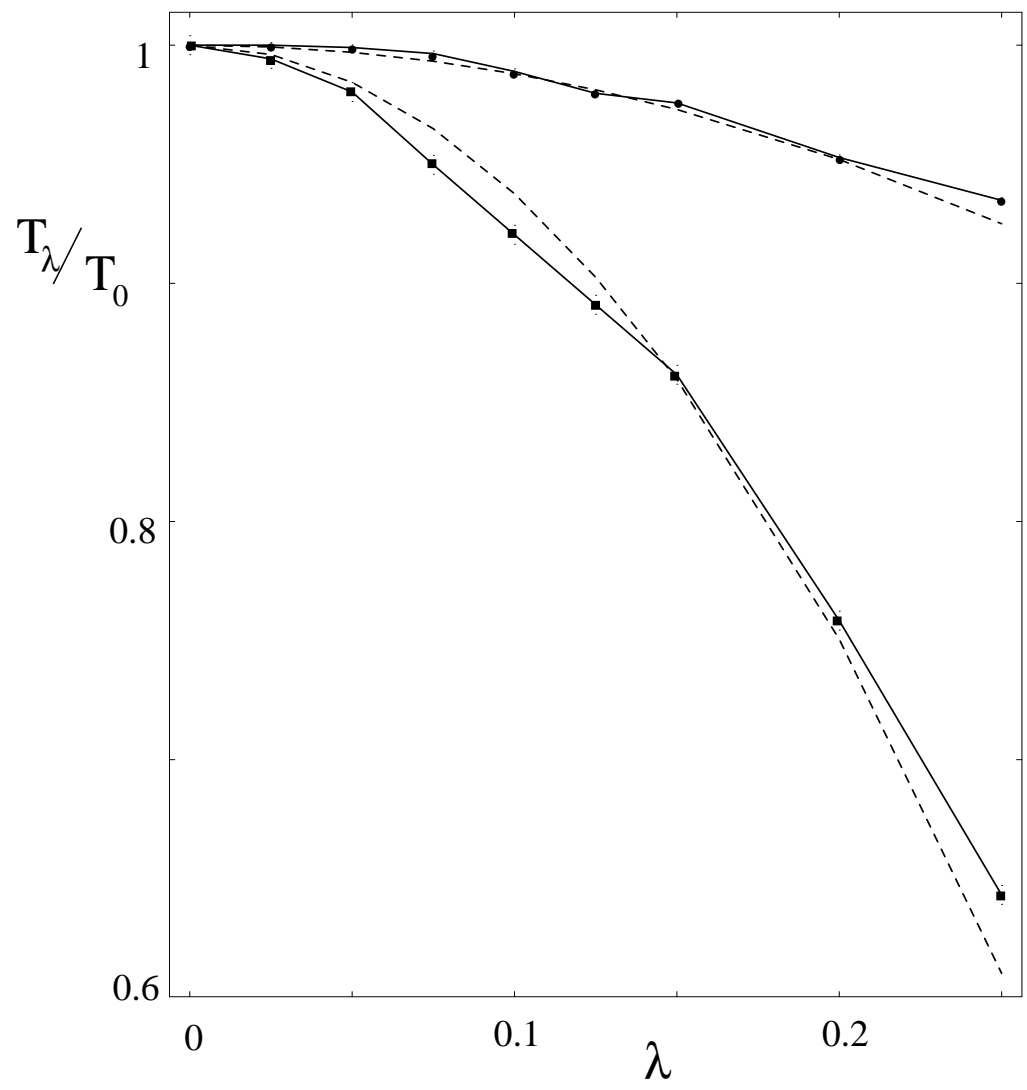

